\title{
Performance of BAT Algorithm on Localization of Wireless Sensor Network
}

\author{
Sonia Goyal, Manjeet Singh Patterh \\ Assistant Professor, Department of Electronics and Communication Engineering \\ University College of Engineering \\ Punjabi University, Patiala (PB), India 144011 \\ Email: goyalsonia_82@yahoo.co.in \\ Professor, Department of Electronics and Communication Engineering \\ University College of Engineering \\ Punjabi University, Patiala (PB), India 144011 \\ Email:mspattar@yahoo.com
}

\begin{abstract}
Many applications of wireless sensor networks (WSN) require information about the geographical location of each sensor node. Devices that form WSN are expected to be remotely deployed in large numbers in a sensing field, and to selforganize to perform sensing and acting task. The goal of localization is to assign geographical coordinates to each device with unknown position in the deployment area. Recently, the popular strategy is to apply optimization algorithms to solve the localization problem. In this paper, the bat algorithm is implemented to estimate the sensor's position.
\end{abstract}

\section{Indexing terms}

Wireless sensor network, localization, Bat algorithm

\section{Academic Discipline And Sub-Disciplines}

Electronics and Communication and Wireless Sensor Network

\section{SUBJECT CLASSIFICATION}

Wireless Sensor Network

\section{TYPE (METHOD/APPROACH)}

Bat Algorithm

\section{Council for Innovative Research}

Peer Review Research Publishing System

\section{Journal: INTERNATIONAL JOURNAL OF COMPUTERS \& TECHNOLOGY}

\author{
Vol 6, No 3 \\ editor@cirworld.com \\ www.cirworld.com, member.cirworld.com
}




\section{INTRODUCTION}

Recent advances in radio and embedded systems have enabled the proliferation of wireless sensor networks. Wireless sensor networks are tremendously being used in different environments to perform various monitoring tasks such as search, rescue, disaster relief, target tracking and a number of tasks in smart environments. In many such tasks, node localization is inherently one of the system parameters. Node localization is required to report the origin of events, assist group querying of sensors, routing and to answer questions on the network coverage. So, one of the fundamental challenges in wireless sensor network is node localization [1].

In a sensor network, there will be a large number of sensor nodes densely deployed at positions which may not be predetermined. In most sensor network applications, the information gathered by these micro-sensors will be meaningless unless the location from where the information is obtained is unknown. This makes localization capabilities highly desirable in sensor networks [2]. Theoretically, a localization measurement device such as GPS can be used for a sensor to locate itself. However, it is not practical to use GPS in every sensor node because a sensor network consists of thousands of nodes and GPS will be very costly. On the other hand, GPS does not work at all in indoor environments, so alternative solutions must be employed [3]. To solve the problem, many localization methods have been developed. Instead of requiring every node to have GPS installed, all localization methods assume only a few nodes be equipped with GPS hardware. These nodes are often called anchor nodes and they know their positions. Other normal sensors can communicate with a few nearby sensors and estimate distances between them using some localization algorithm (e.g. RSS, ToA) and then derive their positions based on the distances[3][4][5].

This paper proposes the application of BAT algorithm for distributed iterative node localization in WSNs. BAT algorithm performs quite well in terms of nodes localized and localization accuracy.

The rest of the paper is organized as follows: Literature Survey on WSN Localization is presented in Section II. Section III the gentle overview of BAT algorithm used for localization in this study. This is followed with implementation of above said algorithm in section IV. Section V presents simulation results. Finally, section VI presents conclusions and makes a projection on possible future research path.

\section{LITERATURE SURVEY}

A detailed survey of the relevant literature is available in [6], [7], [8], [9]. An efficient localization system with Accurate Positioning System (APS) extends the GPS capabilities to non-GPS nodes in ad hoc networks as anchors flood their location information to all nodes in the networks proposed in [10].Then each target node performs a triangulation to three or more anchors to find its position. Node localization accuracy is improved by measuring anchor distances from their neighbors by introducing a refinement phase [11]. The issue of error accumulation is addressed in [12] through Kalman filter based least square estimation in [13] [14] to simultaneously locate the position of all sensor nodes. Node localization problem is addressed using convex optimization based on semi-definite programming. The semi-definite programming approach is further extended to non-convex inequality constraints. In [15] [16], the gradient search technique demonstrates the use of a data analysis technique called multi dimensional scaling (MDS) in estimating the position of unknown nodes. The algorithm localizes an individual patch by first computing all pair wise shortest paths between sensors in the patch. Then it applies MDS to these distances to get an initial layout. Finally, an absolute map is obtained by using the known node positions. These techniques work well with few anchors and reasonably high connectivity.

Soft computing plays a crucial role in optimization problems. WSN is treated as multi-model and multidimensional optimization problem and addressed through population based stochastic techniques. A few GA based node localization algorithms are presented in [17] [18] [19] that estimate optimal node locations of all one-hop neighbors. A two phase centralized localization scheme that uses SAA and GA is presented in [20]. PSO based algorithm is proposed in [21] [22] to minimize the localization error. In [23] two intelligent localization schemes for WSNs are introduced for range-free localization, which utilize received signal strength (RSS) from the anchor nodes. In the first scheme, the edge weight of each anchor node is separately calculated and combined to compute the location of sensor nodes. The edge weights are modeled by a Fuzzy Logic System (FLS) and optimized by the GA. In the second scheme, the localization is approximated as a single problem where the entire sensors locations from the anchor node signals are mapped by a Neural Network (NN) [24]. A two objective evolutionary algorithm which takes concurrently into account, during the evolutionary process, both the localization accuracy and certain topological constraints induced by connectivity considerations using meta heuristic approach, namely SA, is proposed in [25]. Each target node is localized under imprecise measurement of distances from three or more neighboring anchors/settled nodes. computer science. In this paper, BAT algorithm is described in order to evaluate the precision of node localization problem in wireless sensor networks, and the experimental results show that the proposed method is a good solution for node localization evaluation.

\section{BAT ALGORITHM}

Recently, a new meta heuristic search algorithm, called bat algorithm (BA), has been developed by Xin-She Yang (2010a) [26] is explained in section 3 for the convenience of the readers. Preliminary studies show that it is very promising and could outperform existing algorithms. Bats are fascinating animals. They are the only mammals with wings and they also have advanced capability of echolocation. Micro bats use a type of sonar, called, echolocation, to detect prey, avoid obstacles, and locate their roosting crevices in the dark. These bats emit a very loud sound pulse and listen for the echo that bounces back from the surrounding objects. Their pulses vary in properties and can be correlated with their hunting strategies, depending on the species. 
If idealize some of the echolocation characteristics of microbats, a bat algorithm can be developed. For simplicity, use the following approximate or idealized rules:

a) All bats use echolocation to sense distance, and they also 'know' the difference between food / prey and background barriers in some magical way;

b) Bats fly randomly with velocity $v_{i}$ at position $x_{i}$ with a frequency $f_{\min }$, varying wavelength $\lambda$ and loudness $A_{0}$ to search for prey. They can automatically adjust the wavelength (or frequency) of their emitted pulses and adjust the rate of pulse emission $r \in[0,1]$, depending on the proximity of their target;

c) Although the loudness can vary in many ways, we assume that the loudness varies from a large (positive) $A_{0}$ to a minimum constant value $A_{\text {min. }}$.

Based on these approximations and idealization, the basic steps of the Bat Algorithm (BA) [26] can be summarized as the pseudo code shown in Figure 1 as follows.

Fig. 1: Pseudo code of the bat algorithm (BA)

1. Objective function $f(x), x=(x 1, \ldots \ldots x d) T$

2. Initialize the bat population $x_{i}=(i=1,2 \ldots n)$ and $v_{i}$

3. Define Pulse frequency $f_{i}$ at $x_{i}$

4. Initialize the rates $r_{i}$ and the loudness $A_{i}$

5. While $(\mathrm{t}<$ Max number of iterations)

6. Generate new solutions by adjusting frequency, by adjusting frequency, and updating velocities and locations/solutions

7. If $\left(\right.$ rand $\left.>r_{i}\right)$

8. Select a solution among the best solutions

9. Generate a local solution around the selected best solution

10. End if

11. Generate a new solution by flying randomly

12. If $\left(\right.$ rand $\left.<A_{i} \& f\left(x_{i}\right)<f\left(x^{*}\right)\right)$

13. Accept the new solutions

14. Increase $r_{i}$ and reduce $A_{i}$

15. End if

16. Rank the bats and find the current best $x^{*}$

17. End while

18. Post process results and visualization

Another obvious simplification is that no ray tracing is used in estimating the time delay and the three dimensional topography. Though this might be a good feature for the application in computational geometry, however, we will not use this as it is more computationally extensive in multidimensional cases. In addition to these simplified assumptions, we also use the following approximations, for simplicity. In general the frequency $f$ in a range $\left[f_{\min }, f_{\max }\right]$ corresponds to a range of wavelengths $\left[\lambda_{\min }, \lambda_{\max }\right]$. For example a frequency range of $[20 \mathrm{kHz}, 500 \mathrm{kHz}]$ corresponds to a range of wavelengths from $0.7 \mathrm{~mm}$ to $17 \mathrm{~mm}$.

\subsection{Bat Motion}

For the bats in simulations, we have to define the rule show their positions $x_{i}$ and velocities $v_{i}$ in a d-dimensional search space are updated. The new solutions $x_{i}^{t}$ and velocities $v_{i}^{t}$ at time step $t$ are given by

$$
f_{i}=f_{\min }+\left(f_{\max }-f_{\min }\right) \beta
$$




$$
\begin{gathered}
v_{i}^{t+1}=v_{i}^{t}+\left(x_{i}^{t}-x^{*}\right) f_{i}, \\
x_{i}^{t+1}=x_{i}^{t}+v_{i}^{t},
\end{gathered}
$$

where $\beta \in[0,1]$ is a random vector drawn from a uniform distribution. Where $x^{*}$ is a current global best location (solution) which is located after comparing all the solutions among all the $\mathrm{n}$ bats at each iterations $\mathrm{t}$. Initially, each bat is randomly assigned a frequency which is drawn uniformly from $\left[f_{\min }, f_{\max }\right]$.

The update of the velocities and positions of bats have some similarity to the procedure in the standard particle swarm optimization, as $f_{i}$ essentially controls the pace and range of the movement of the swarming particles. To a degree, BA can be considered as a balanced combination of the standard particle swarm optimization and the intensive local search controlled by the loudness and pulse rate.

\subsection{Loudness and Pulse Emission}

Furthermore, the loudness $A_{i}$ and the rate $r_{i}$ of pulse emission have to be updated accordingly as the iterations proceed. As the loudness usually decreases once a bat has found its prey, while the rate of pulse emission increases, the loudness can be chosen as any value of convenience [27].

\section{BAT ALGORITHM BASED LOCALIZATION}

The main objective in WSN localization is to find out the coordinate of maximum number of target nodes by using anchor nodes with single hop range-based distributed technique. To estimate coordinates of $\mathrm{N}$ target nodes, the process followed is as below:

1) $N$ Target nodes and $M$ anchor nodes are randomly deployed in the sensor field. Each target node and anchor node has transmission range R. Anchor nodes compute their location awareness and transmit their coordinates. The nodes, which get settled at the end of iteration, serve as reference nodes during the next iteration and behave like anchors.

2) The node that falls with in transmission range of three or more anchors is considered as localized node.

3) Each localized node measures its distance from each of its neighboring anchors. The distance measurements are corrupted with Gaussian noise $n_{i}$, due to environment consideration. A node estimates its distance from ith anchor as $d$ $=\left[d_{i}+n_{i}\right]$ where $d$ is actual distance given by

$$
\mathrm{d}_{\mathrm{i}}=\sqrt{\left(\mathrm{x}-\mathrm{x}_{i}\right)^{2}+\left(\mathrm{y}-\mathrm{y}_{i}\right)^{2}}
$$

whereas $(x, y)$ is the location of the target node and $\left(x_{i}, y_{i}\right)$ is the location of the ith anchor node in the neighborhood.

4) Each localizable target node runs BAT algorithm independently to localize itself by finding its coordinates ( $x, y)$. The objective function (mean of square of error between actual and estimated distances of computed node coordinates and the actual node coordinates), given as below is minimized.

$$
\mathrm{f}(\mathrm{x}, \mathrm{y})=1 / \mathrm{M} \sum_{i=1}^{M}\left(\sqrt{\left(\mathrm{x}-\mathrm{x}_{i}\right)^{2}+\left(\mathrm{y}-\mathrm{y}_{i}\right)^{2}}-\mathrm{d}_{\mathrm{i}}\right)
$$

where $\mathrm{M} \geq 3$ ( $2 \mathrm{D}$ location of a node needs minimum 3 anchors) is the number of anchors with in transmission range, $\mathrm{R}$, of the target node.

5) BAT evolve the optimal location of target nodes, i.e. $(x, y)$ by minimizing the error function

6) After coordinate so fall localizable nodes (say, $N_{L}$ ) are determined, the mean localization error is computed as the mean of square of distance so of computed node coordinates $\left(x_{i} y_{i}\right)$ and the actual node coordinates $\left(X_{i} Y_{i}\right)$, for $i=1,2, \ldots . . N_{L}$ determined for BAT, as shown below:

$$
\mathrm{MLE}=\frac{\sum_{i=M+1}^{N} \sqrt{\left(\mathrm{x}_{i}-\mathrm{X}_{i}\right)^{2}+\left(\mathrm{y}_{i}-\mathrm{Y}_{\mathrm{i}}\right)^{2}}}{\left(\mathrm{~N}_{\mathrm{L}}\right) \mathrm{R}}
$$

7) Steps 2 to 6 are repeated until all target nodes get localized or no more nodes can be localized. The performance of the localization algorithm is based on MLE and $N_{N L}$, where $N_{N L}=\left[N-N_{L}\right]$ is the number of nodes that could not be localized. Lesser the value of $\mathrm{N}_{\mathrm{NL}}$ and MLE, better the performance is.

As the iterations progress, the number of localized nodes increases. This increases the number of references available for already localized nodes. A node that localizes using just three references in an iteration $\mathrm{k}$ may have more references in iteration $\mathrm{k}+1$. This decreases the probability of the flip ambiguity. 


\section{SIMULATION RESULTS AND DISCUSSION}

The WSN localization simulations were conducted using BAT algorithm in MATLAB environment. N target nodes and $10 \%$ anchor nodes are randomly deployed in a sensor field. The physical dimension $\mathrm{R}$ of the square area for spreading the sensors is, $R=1.2 r, 1.6 r$, 2.0r where $r$ denotes the predetermined communication range of each sensor node. Other strategic settings are specific to BAT algorithm as discussed below:

In the proposed frame work, BAT parameters for node localization are fixed as shown in Table 1.

Table 1.The parameters value of experiment

\begin{tabular}{|c|c|}
\hline Parameters & Parameters value \\
\hline Population size & 2 \\
\hline Number of generations & 100 \\
\hline Loudness & 0.5 \\
\hline Pulse rate & 0.5 \\
\hline Minimum Frequency & 0 \\
\hline Maximum Frequency & 2 \\
\hline
\end{tabular}

BAT algorithm is stochastic in nature; so one can't expect the same solution in all trials with identical deployment. This is the reason why the results of many trial runs are averaged. The initial deployment is random, so, the number of localizable nodes in each iteration is not expected to be the same. The sensor nodes position is calculated using BA. The true and estimated sensor locations are shown in the Figure 2.

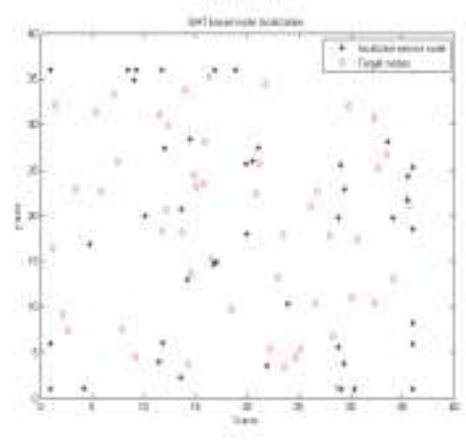

Fig. 2: Node Localization with BAT algorithm

Mean Localization Error and number of localized nodes are depending upon four parameters: Field size, transmission range, number of anchor nodes and total number of sensor nodes.

\subsection{Mean localization error vs. field size}

The results based on BA are summarized in Table 2. Each point in the simulation results after 30 repetitions of experiments performed on 30 independent configurations. Table II shows with the increase of field size the numbers of localized nodes is decreasing and mean localization error is increasing. But it gives the optimum value of localized nodes and mean localization error at $\mathrm{R}=2.0 \mathrm{r}$.

Table 2. Summary of iterative simulation results based on BA

Anchor Nodes $=12$, Transmission range $(r)=30$, No. of target nodes $=40$, $R=$ Field size

$\mathrm{NL}=$ Localized nodes, MLE=Mean localization error

\begin{tabular}{l|cc|ccccr}
\multicolumn{2}{c|}{ Iterations } & 1 & \multicolumn{1}{c|}{2} & 3 & 4 & \multicolumn{1}{c}{5} & \multicolumn{1}{c}{ Avg } \\
$\mathrm{R}=1.2 \mathrm{r}$ & $\mathrm{NL}$ & 40 & 40 & 40 & 40 & 40 & 40 \\
& $\mathrm{MLE}$ & 0.53 & 0.60 & 0.53 & 0.56 & 0.65 & 0.574 \\
$\mathrm{R}=1.4 \mathrm{r}$ & $\mathrm{NL}$ & 40 & 40 & 40 & 40 & 40 & 40 \\
& $\mathrm{MLE}$ & 0.59 & 0.65 & 0.67 & 0.69 & 0.69 & 0.658 \\
$\mathrm{R}=1.6 \mathrm{r}$ & $\mathrm{NL}$ & 40 & 40 & 40 & 40 & 40 & 40 \\
& $\mathrm{MLE}$ & 0.70 & 0.82 & 0.76 & 0.79 & 0.73 & 0.76
\end{tabular}




\begin{tabular}{l|cr|rrrrr}
$\mathrm{R}=1.8 \mathrm{r}$ & $\mathrm{NL}$ & 40 & 40 & 39 & 39 & 39 & 39 \\
& $\mathrm{MLE}$ & 0.79 & 0.79 & 0.67 & 0.76 & 0.64 & 0.73 \\
$\mathrm{R}=2.0 \mathrm{r}$ & $\mathrm{NL}$ & 40 & 39 & 39 & 39 & 39 & 39 \\
& $\mathrm{MLE}$ & 0.51 & 0.64 & 0.62 & 0.67 & 0.73 & 0.634 \\
$\mathrm{R}=2.2 \mathrm{r}$ & $\mathrm{NL}$ & 37 & 38 & 38 & 37 & 38 & 38 \\
& $\mathrm{MLE}$ & 0.67 & 0.60 & 0.63 & 0.69 & 0.67 & 0.652
\end{tabular}

\subsection{Mean Localization Error vs. Transmission range}

To allow for easy comparison between different scenarios, errors in location estimates are normalized by the transmission range. For every set of simulations, the numbers of nodes are fixed and the transmission range is varied. The mean localization error as a function of the transmission range is shown in the Figure 3 . Figure shows as with the increase of transmission range mean localization error is decreasing.

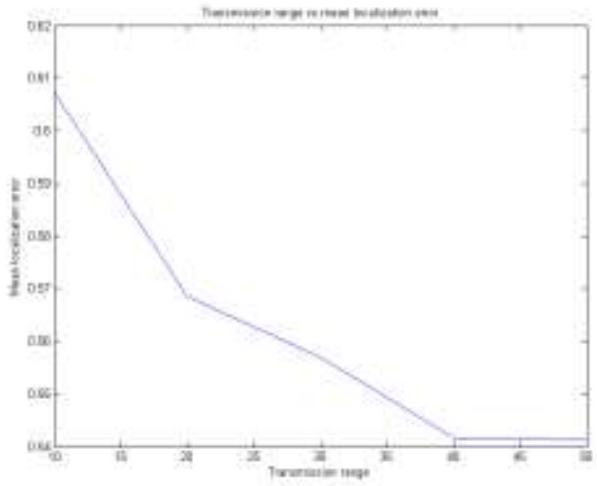

Fig. 3: Mean Localization Error vs. Transmission range

\subsection{Mean Localization Error vs. Anchor nodes}

The mean localization error is further reduced as we increase the number of anchor nodes as shown in Figure 4 where anchor nodes are varying like $10 \%, 20 \%, 30 \%, 40 \%$ and $50 \%$.
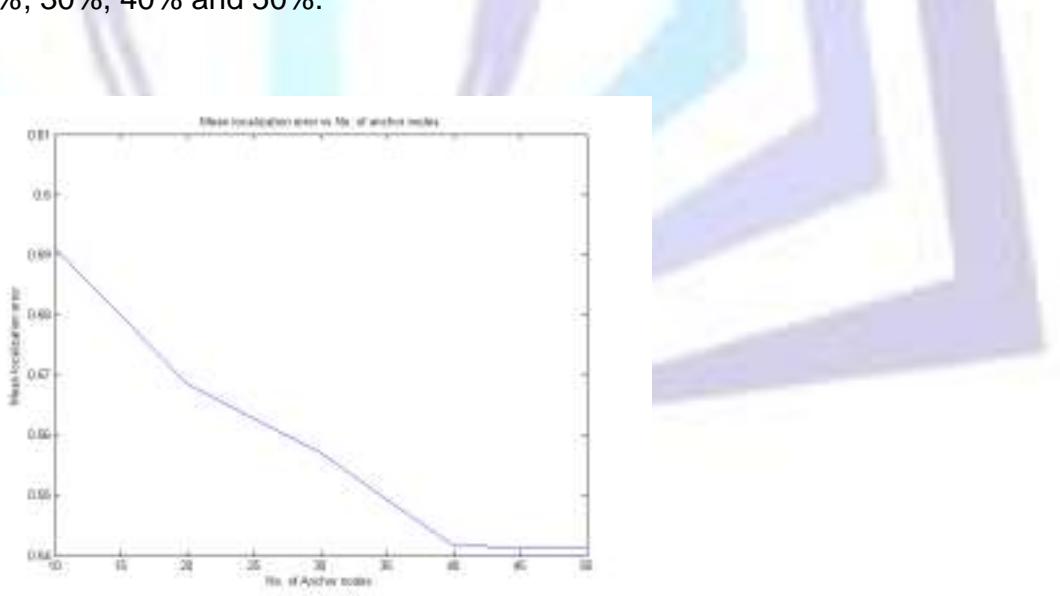

Fig. 4: Mean Localization Error vs. No. of Anchor nodes

\subsection{Mean Localization Error vs. No. of sensor Nodes}

Mean localization error also depends upon number of sensor nodes. As number of nodes is increasing the MLE also goes on increasing as shown in Figure 5. 


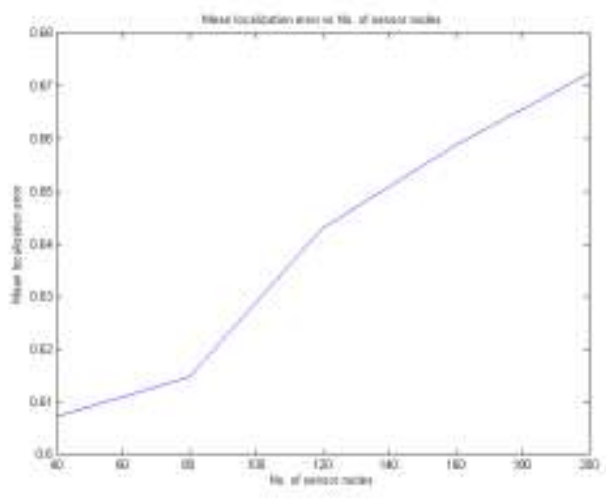

Fig. 5: Mean Localization Error vs. No. of sensor nodes.

\section{CONCLUSION}

Localization is one of the key techniques of wireless sensor networks. In this paper the localization problem in WSN is formulated as an optimization problem and Bat algorithm is utilized to solve this problem. The simulation result shows that the mean localization error goes on decreasing as we increase the number of anchor nodes and transmission range and it is increasing as we increase the number of sensor nodes and field size. A hybrid stochastic algorithm may be proposed to achieve more accuracy. The effectiveness of proposed algorithm may verify on experimental set up of sensor network.

\section{ACKNOWLEDGMENTS}

Our thanks to the Dr. Lakhwinder Kaur for her timely and carefully help.

\section{REFERENCES}

[1] Amitangshu Pal , "Localization Algorithms in Wireless Sensor Networks: Current Approaches and Future Challenges," Network Protocols and Algorithms ISSN 1943-3581 2010, Vol. 2, No. 1

[2] Anushiya A Kannan, Guoqiang Mao and Branka Vucetic, " Simulated Annealing based Wireless Sensor Network Localization" Journals of computer, vol 1, No. 2, May 2006.

[3] L. Doherty et al., "Convex position estimation in wireless sensor networks," in INFOCOM 2001. Twentieth Annual Joint Conference of the IEEE Computer and Communications Societies. Proceedings., vol.3, 2001, pp. 1655-1663.

[4] R. Kulkarni, G. Venayagamoorthy, and M. Cheng, "Bio-inspired node localization in wireless sensor networks," in IEEE International Conference on Systems, Man and Cybernetics, 2009. SMC 2009., pp. 205-210.

[5] A. Pal, "Localization algorithms in wireless sensor networks: Current approaches and future challenges," Network Protocols and Algorithms, vol. 2, no.1, pp.45-73, 2010.

[6] I. Akyildiz, W. Su, Y. Sankarasubramaniam, and E. Cayirci, "Wireless sensor networks : a survey," Computer networks, vol. 38, no. 4, pp. 393-422, 2002.

[7] J. Wang, R. K. Ghosh, and S. K. Das, "A survey on sensor localization," Journal of Control Theory and Applications, vol. 8, no. 1, pp. 2-11, 2010.

[8] A. Boukerche, H. Oliveira, E. Nakamura, and A. Loureiro, "Localization systems for wireless sensor networks," wireless Communications, IEEE, vol. 14, no. 6, pp. 6-12, 2007.

[9] J. Hightower and G. Borriello, "Location systems for ubiquitous computing," Computer, vol. 34, no. 8, pp.57-66, 2001.

[10] D. Niculescu and B. Nath, "Ad hoc positioning system (aps)," in Global Telecommunications Conference, 2001. GLOBECOM 01. IEEE, vol. 5. IEEE, 2001, pp. 2926-2931.

[11] N. Bulusu, D. Estrin, L. Girod, and J. Heidemann, "Scalable coordination for wireless sensor networks: selfconfiguring localization systems," in International Symposiumon Communication Theory and Applications (ISCTA2001), Ambleside, UK, 2001.

[12] A. Savvides, H. Park, and M. Srivastava, " The bits and flops of the n-hop multilateration primitive for node localization problems," in Proceedings of the $1^{\text {st }}$ ACM international workshop on Wireless sensor networks and applications. ACM, 2002, pp. 112-121.

[13] M. Di Rocco and F. Pascucci, "Sensor network localization using distributed extended kalman filter," in IEEE/ASME international conference on Advanced intelligent mechatronics, 2007. IEEE, 2007, pp.1-6. 
[14] R. Kalman, "A new approach to linear filtering and prediction problems," Journal of basic Engineering, vol. 82, no. Series D, pp. 35-45, 1960.

[15] Y. Shang and W. Ruml, "Improved MDS-based localization," in Twenty third Annual Joint Conference of the IEEE Computer and Communications Societies INFOCOM 2004, vol. 4. IEEE, 2004, pp. 2640-2651.

[16] P. Biswas, T. Lian, T. Wang, and Y.Ye, "Semi definite programming based algorithms for sensor network localization," ACM Transactions on Sensor Networks (TOSN), vol. 2, no. 2, pp. 188-220, 2006.

[17] S. Yun, J. Lee, W. Chung, E. Kim, and S. Kim, "A soft computing approach to localization in wireless sensor networks," Expert Systems with Applications, vol. 36, no. 4, pp.7552-7561,2009.

[18] Q. Zhang, J. Wang, C. Jin, and Q. Zeng, "Localization algorithm for wireless sensor network based on genetic simulated annealing algorithm," in $4^{\text {th }}$ International Conference on Wireless Communications Networking and Mobile Computing, 2008. WiCOM08. IEEE, 2008, pp.1-5.

[19] Q. Zhang, J. Huang, J. Wang, C. Jin, J. Ye, and W. Zhang, "A new centralized localization algorithm for wireless sensor network," in Third International Conference on Communications and Networking in China, physical world with pervasive networks," Pervasive Computing, IEEE, 2008. China Com 2008. IEEE, 2008, pp. 625-629.

[20] Y. Li, J. Xing, Q. Yang, and H. Shi, "Localization research based on improved simulated annealing algorithm in WSN," in $5^{\text {th }}$ International Communications of Conference on Wireless Communications, Networking and Mobile Computing, 2009. WiCom 09. IEEE, 2009, pp. 1-4.

[21] R. Kulkarni, G. Venayagamoorthy, and M. Cheng, "Bio-inspired node localization in wireless sensor networks," in IEEE International Conference on Systems, Man and Cybernetics, 2009. SMC 2009., pp.205-210.],

[22] A. Gopakumar and L. Jacob, "Localization in wireless sensor networks using particle swarm optimization," in IET International Conference on Wireless, Mobile and Multimedia Networks, 2008. IET, 2008, pp.227-230.

[23] R. Stoleru and J. A. Stankovic, "Probability grid: A location estimation scheme for wireless sensor networks," in First Annual IEEE Communications Society Conference on Sensor and Ad Hoc Communications networks, 2004. IEEE SECON 2004. IEEE, 2004, pp.430-438.

[24] P. Chuang and C. Wu, "An effective pso-based node localization scheme for wireless sensor networks," in Ninth International Conference on Parallel and Distributed Computing, Applications and Technologies, 2008. PDCAT 2008. IEEE, 2008, pp.187-194.

[25] G. Mao, B. Fidan, and B. Anderson, "Wireless sensor network localization techniques," Computer Networks, vol. 51, no.10, pp.2529-2553, 2007.

[26] Yang, X. S. (2010a). A new metaheuristic bat-inspired algorithm, in: Nature Inspired Cooperative Strategies for Optimization (NICSO2010) (Eds. J. R. Gonzalez et al.), Springer, SCI Vol. 284, 65-74.

[27] Yang, X. S., (2011), Bat Algorithm for Multiobjective Optimization, Int. J. Bio-Inspired Computation, Vol. 3, No. 5, pp. 267-274.

\section{Author' biography}

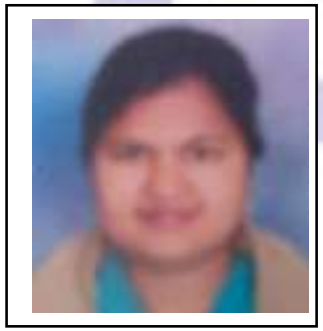

Sonia Goyal is received the B.Tech and M.E degree in engineering from Punjab Technical University, Jalandhar, India and Punjab University, Chandigarh, India respectively She has been in teaching for the last 08 years. Presently she is Assistant Professor at University College of Engineering, Punjabi University, Patiala. She is pursuing P.hd from Punjabi university, Patiala.She has to her credit many papers in international journals and national and international conferences.

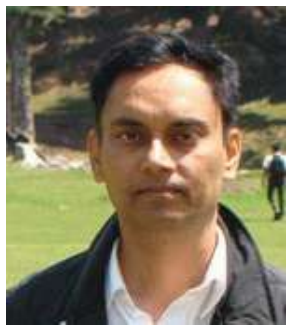

Manjeet Singh Patterh, did his Bachelor's degree from Madhav Institute of Technology and Science (MITS), Gwalior (MP) and Master's degree from Birla Institute of Technology and Science (BITS), Pilani, both in Electronics Engineering. He did his PhD from Punjab Technical University Jalandhar. He has 60 publications in international, national refereed journals and conferences. He is having over 23 years of teaching experience. He is presently working as Professor in department of electronics and communication engineering at Punjabi University Patiala. His current interests are Digital Signal Processing, Wireless Communication Systems and Networking. He is member of IEEE and life member of ISTE, IE (I) and IETE 